\title{
The Unmet Need for Postacute Rehabilitation Among Medicare Observation Patients: A Single-Center Study
}

\author{
Jennifer N. Goldstein, MD, MSc ${ }^{1,2 \star}$, J. Sanford Schwartz, MD³, Patricia McGraw, RN¹, Tobias L. Banks, DOํ, LeRoi S. Hicks, MD, MPH',2 \\ ${ }^{1}$ Department of Medicine and ${ }^{2}$ Value Institute, Christiana Care Health System, Newark, Delaware; ${ }^{3}$ Division of General Internal Medicine, University \\ of Pennsylvania, Philadelphia, Pennsylvania.
}

BACKGROUND: Medicare beneficiaries admitted under observation status must pay for postacute inpatient rehabilitation (PAIR) services, out of pocket, at potentially prohibitive costs.

OBJECTIVE: To determine if there is an unmet need for PAIR among Medicare observation patients and if this care is associated with longer hospital stay and increased rehospitalization.

DESIGN/SETTING: Observational study using electronic medical record and administrative data from a regional health system.

PATIENTS: 1323 community-dwelling Medicare patients admitted under observation status.

MEASUREMENTS: Summary statistics were calculated for demographic and administrative variables. Physical therapy (PT) and case management recommendations for a representative sample of 386 medical records were reviewed regarding need for PAIR services. Linear regression was used to measure the association between $\mathrm{PT}$ recommendation and hospital length of stay, adjusting for ICD-9 (International Classification of Diseases, Ninth Revision) diagnosis, age, sex, and provider. Chi-square test was used to determine the association between PT recommendation and 30-day hospital revisit.

RESULTS: Of the 1323 study patients, 11 (0.83\%) were discharged to PAIR facilities. However, 17 (4.4\%) of the 386 patients whose charts were reviewed received a recommendation for this care. Adjusted mean hospital stay was longer $(P$ $<0.001)$ for patients recommended for rehabilitation $(75.9 \mathrm{~h})$ than for patients with no PT needs $(46.8 \mathrm{~h})$. In addition, the 30 -day hospital revisit rate was higher $(P=0.037)$ for the patients who had been recommended for rehabilitation $(52.9 \%$, 9/17) than for those who had not (25.4\%, 30/118).

CONCLUSIONS: Medicare observation patients' potential need for PAIR services is 5- to 6-fold higher than their use of these services. Observation patients recommended for this care may have worse outcomes. Journal of Hospital Medicine 2017;12:168-172. (C) 2017 Society of Hospital Medicine.
As the US population ages and becomes increasingly frail, the need for rehabilitation rises. By 2030, an estimated $20 \%$ of the population will be 65 years old or older, and almost $10 \%$ will be over $75 .^{1}$ About $20 \%$ of hospitalized Medicare patients receive subsequent care in postacute inpatient rehabilitation (PAIR) facilities, accounting for $\$ 31$ billion in Medicare expenditures in $2014 .^{2}$ Although the need for rehabilitation will continue to rise, Medicare policy restricts access to it.

Under Medicare policy, PAIR services are covered for certain hospitalized patients but not others. Hospitalized patients are either inpatients, who are billed under Medicare Part A, or outpatients, billed under Part B. When hospital length of stay (LOS) is anticipated to be less than 2 midnights, patients are admitted as outpatients under the term observation status; when longer stays are expected, patients

\footnotetext{
*Address for correspondence and reprint requests: Jennifer N. Goldstein, MD, MSc, Department of Medicine, Christiana Care Health System, Suite 2E70, Ammon Education Building, 4755 Ogletown-Stanton Rd, Newark, DE 19713; Telephone: 302-733-6591; Fax: 302-733-6082; E-mail: jgoldstein@ christianacare.org

Additional Supporting Information may be found in the online version of this article.

Received: June 8, 2016; Revised: August 22, 2016; Accepted: September 11, 2016
}

2017 Society of Hospital Medicine DOI 10.12788/jhm.2700 are admitted as inpatients. ${ }^{3}$ This recently implemented time-based distinction has been criticized as arbitrary, and as potentially shifting many patients from inpatient to outpatient (observation) status. ${ }^{4}$

The distinction between inpatient and observation status has significant consequences for posthospital care. Medicare Part A covers care in skilled nursing facilities (SNFs) and acute inpatient rehabilitation facilities (IRFs); after hospitalization, inpatients have access to either, without copay. As observation patients are covered under Medicare Part B, they are technically not covered for either service after their hospital stay. IRFs sometimes accept patients from ambulatory and nonacute settings; observation patients may be accepted in rare circumstances, but they pay the Part A deductible ( $\$ 1288$ in 2016) to have the services covered by Medicare. SNF services are never covered for observation patients, and access to this care requires an average out-ofpocket payment of more than $\$ 10,503$ per beneficiary for a typical SNF stay. ${ }^{5}$ Given that about $70 \%$ of Medicare patients fall below $300 \%$ of the federal poverty line, ${ }^{6}$ the outof-pocket costs for PAIR services for observation patients can be prohibitive.

Although only $0.75 \%$ of community-dwelling Medicare observation patients are discharged to PAIR facilities, ${ }^{7}$ it is unclear if the need for this care is higher but remains unmet secondary to cost concerns of Medicare beneficiaries. Also 
unclear is whether observation patients who would benefit from this care but do not receive it end up with poorer health outcomes and therefore use more healthcare services.

The purpose of this study was to estimate the proportion of Medicare observation patients who are admitted from home and receive a recommendation for placement in a PAIR facility, and to determine the ultimate disposition of such patients. We also sought to evaluate the association between recommendation for PAIR placement, LOS, and 30-day hospital revisit rate.

\section{METHODS}

The Institutional Review Board of Christiana Care Health System (CCHS) approved this study.

\section{Sample and Design}

This was an observational study of community-dwelling Medicare patients admitted under observation status to Delaware's CCHS, which consists of a 907-bed regional tertiary-care facility in Newark and a 241-bed community hospital in Wilmington. The study period was January 1 to December 31,2013 . We limited our sample to patients treated by hospitalists on hospital wards, as this care constitutes $80 \%$ of the care provided to observation patients at CCHS and the majority of care nationally. ${ }^{8}$ As neither SNF care nor IRF care is covered under Medicare Part B, and both would result in high out-of-pocket costs for Medicare observation patients, we combined them into a single variable, PAIR.

All data were obtained from institutional electronic medical record and administrative data systems. Study inclusion criteria were Medicare as primary insurance, admission to hospital from home, and care received at either CCHS facility. Exclusion criteria were admission from PAIR facility, longterm care facility, assisted-living facility, or inpatient psychiatric facility; death; discharge against medical advice (AMA) or to hospice, non-SNF, or inpatient psychiatric facility; and discovery (during review of case management [CM] notes) of erroneous listing of Medicare as primary insurance, or of inpatient admission (within 30 days before index observation stay) that qualified for PAIR coverage under Medicare Part A.

We reviewed the medical charts of a representative ( 30\%) sample of the cohort and examined physical therapy (PT) and $\mathrm{CM}$ notes to determine the proportions of patients with recommendations for home with no services, home-based PT, possible PAIR, and PAIR. Charts were sorted by medical record number and were reviewed in consecutive order. We coded a patient as having a recommendation for possible PAIR if the PT notes indicated the patient may benefit from PAIR but could have home PT if PAIR placement was not possible. $\mathrm{CM}$ notes were also reviewed for evidence of patient or family preference regarding PAIR placement. All questions about PT and $\mathrm{CM}$ recommendations were resolved by consensus.

\section{Measures}

For the total study sample, we calculated descriptive statistics and frequencies for demographic and administrative variables, including age, sex, race (Caucasian, African American, other), ethnicity (Hispanic/non-Hispanic), ICD-9 (International Classification of Diseases, Ninth Revision) primary diagnosis code, LOS (in hours) for index observation admission, discharge disposition (home with no services, home PT, possible PAIR, PAIR), and 30-day hospital revisit (emergency department, observation, inpatient admission). We used $\chi^{2}$ test, Student $t$ test, and analysis of variance (ANOVA) to test for statistically significant differences in characteristics between the chart review subgroup and the rest of the sample and between the groups with different disposition recommendations from PT notes.

For the chart review subgroup, we used ANOVA to calculate the unadjusted association between PT recommendation and LOS. We then adjusted for potential confounders, using multivariable linear regression with PT recommendation as a predictor and LOS as the outcome, controlling for variables previously associated with increased LOS among observation patients (primary diagnosis category, age, sex). ${ }^{6}$ We also adjusted for hospitalist group to account for potential variability in care delivery. As LOS was not normally distributed, we calculated the fourth root of LOS, which resulted in a more normal distribution, and used the transformed values in the regression model. We then calculated predicted values from the regression and back-transformed these to obtain adjusted mean values for LOS.

\section{RESULTS}

Of the 1417 unique patients who had Medicare as primary insurance and were admitted under observation status to a hospitalist service during the study period (2013), 94 were excluded (Figure). Of the remaining 1323 patients, the majority were 65 years old or older, female, white, and non-Hispanic. The most common ICD-9 diagnoses were syncope and chest pain. Mean LOS was 46.7 hours (range, 0-519 h). Less than $1 \%$ of patients were discharged to PAIR. Almost $25 \%$ of patients returned to the hospital, either for an emergency department visit or for observation or inpatient stay, within 30 days (Table).

Of the 419 charts reviewed to determine the proportion of patients evaluated by PT, and their subsequent recommendations, 33 were excluded, leaving 386 (92\%) for analysis (Figure). There were no significant demographic differences between the patients in the chart review subgroup and the rest of the patients (Appendix). Of the 386 patients whose charts were analyzed, 181 (46.9\%) had a PT evaluation, and $17(4.4 \%)$ received a PAIR recommendation (Figure). Of the 17 patients recommended for PAIR, $12(70.5 \%)$ were 65 years old or older, and 1 was discharged to a PAIR facility. Of the 46 patients recommended for home PT, 29 (63\%) were discharged home with no services (Table).

PT-evaluated patients had unadjusted mean LOS of 52.2 hours (discharged home with no services), 64.1 hours (home PT or possible PAIR), and 83.1 hours (PAIR) $(P=0.001)$. With adjustment made for variables previously associated with increased LOS for observation patients, mean LOS for 


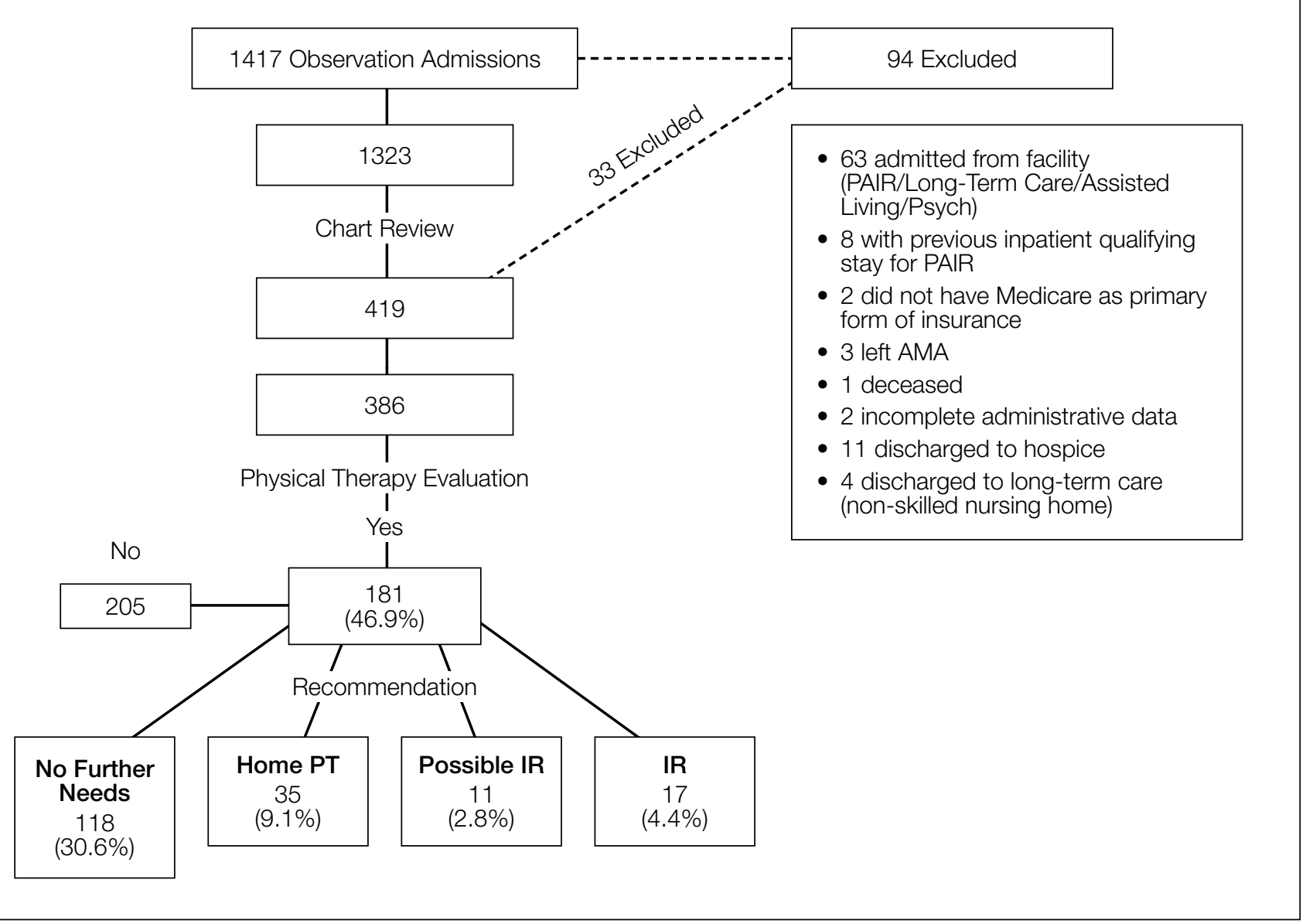

FIG. Selection of study population.

NOTE: Abbreviations: AMA, against medical advice; IR, inpatient rehabilitation; PAIR, postacute inpatient rehabilitation; PT, physical therapy.

patients recommended for PAIR remained higher than that for patients in the other 2 categories (Table). Patients recommended for PAIR were more likely to return to hospital within 30 days than patients recommended for home PT or possible PAIR and patients discharged home with no services (Table).

Review of CM notes revealed that, of the 17 patients recommended for PAIR, 7 would have accepted PAIR services had they been covered by Medicare, 4 preferred discharge with home health services, and 6 did not provide clear details of patient or family preference.

\section{DISCUSSION}

To our knowledge, this is the first study to use chart review to examine the proportion of observation patients who would benefit from PAIR and the relationships among these patients' rehabilitation needs, dispositions, and outcomes. We tried to be conservative in our estimates by limiting the study population to patients admitted from home. Nevertheless, the potential need for PAIR significantly outweighed the actual use of PAIR on discharge. The study sample was consistent with nationally representative samples of observation patients in terms of proportion of patients admitted from and discharged to facilities ${ }^{7}$ and the most common ICD-9 diagnoses. ${ }^{9}$

\section{Physical Therapy Consultations and Observation}

Of the 386 patients whose charts were reviewed and analyzed, 17 (4.4\%) were evaluated as medically qualifying for and potentially benefiting from PAIR. Although the rate represents a minority of patients, it is 5- to 6-fold higher than the rate of discharge to PAIR, both in our study population and in previous national samples that used administrative data. ${ }^{7}$ In some cases, the decision not to discharge the patient to PAIR reflected patient and family preference. However, in other cases, patients clearly could have benefited from PAIR and would have gone had it been covered by Medicare. The gap suggests an unmet need for PAIR among a substantial proportion of Medicare beneficiaries for whom the therapy is recommended and wanted.

Efforts to expand coverage for PAIR have been resisted. According to Medicare regulations, beneficiaries qualify for PAIR coverage if they are hospitalized as inpatients for 3 midnights or longer. Days under observation status do not count toward this requirement, even if this status is changed to inpatient. ${ }^{10}$ The Medicare Payment Advisory Commission (MedPAC) 
TABLE. Characteristics of Study Population and Association of Physical Therapy Recommendations and Outcomes

\begin{tabular}{|c|c|c|c|c|c|c|}
\hline \multirow[b]{2}{*}{ Characteristic } & \multirow[b]{2}{*}{$\begin{array}{l}\text { Total Sample } \\
\qquad(N=1323)\end{array}$} & \multirow[b]{2}{*}{$\begin{array}{l}\text { Patients With } \\
\text { PT Evaluation } \\
\quad(n=181)\end{array}$} & \multicolumn{4}{|c|}{ PT Recommendation $(n=181)$} \\
\hline & & & $\begin{array}{l}\text { Home With } \\
\text { No Services } \\
(n=118)\end{array}$ & $\begin{array}{l}\text { Home PT or } \\
\text { Possible PAIR } \\
\quad(n=46)\end{array}$ & $\begin{array}{c}\text { PAIR } \\
(n=17)\end{array}$ & $P^{a}$ \\
\hline Age, y & & & & & & 0.006 \\
\hline $18-64$ & $21.8 \%(289)$ & $15.5 \%(28)$ & $17.2 \%(21)$ & $4.4 \%(2)$ & $29.4 \%(5)$ & - \\
\hline $65-75$ & $32.9 \%(436)$ & $33.1 \%(60)$ & $38.1 \%(45)$ & $30.4 \%(14)$ & $5.9 \%(1)$ & - \\
\hline Female sex & $64.1 \%(848)$ & $65.7 \%(119)$ & $65.6 \%(78)$ & $25.2 \%(30)$ & $9.2 \%(11)$ & 0.990 \\
\hline Race & & & & & & 0.735 \\
\hline Caucasian & $76.4 \%(1012)$ & $81.2 \%(147)$ & $82.2 \%(97)$ & $82.6 \%(38)$ & $70.6(12)$ & - \\
\hline African American & $20.2 \%(267)$ & $16.6 \%(30)$ & $16.1 \%(19)$ & $15.2 \%(7)$ & $23.5 \%(4)$ & - \\
\hline Other & $3.4 \%(44)$ & $2.2 \%(4)$ & $1.69 \%(2)$ & $2.2 \%(1)$ & $5.9 \%(1)$ & - \\
\hline Chest pain (786.59) & $8.4 \%(111)$ & $3.3 \%(6)$ & $5.1 \%(6)$ & 0 & 0 & - \\
\hline Dizziness/giddiness (780.4) & $4.2 \%(56)$ & $5.5 \%(10)$ & $5.1 \%(6)$ & $8.7 \%(4)$ & 0 & - \\
\hline Urinary tract infection (599) & $2.9 \%(38)$ & $4.4 \%(8)$ & $4.2 \%(5)$ & $1.7 \%(2)$ & $5.9 \%(1)$ & - \\
\hline Altered mental status (780.97) & $2.5 \%(33)$ & $3.3 \%(6)$ & $1.7 \%(2)$ & $2.2 \%(1)$ & $17.6 \%(3)$ & - \\
\hline Discharge disposition & & & & & & - \\
\hline Home with no services & $85.3 \%(1128)$ & $77.3 \%(140)$ & $86.4 \%(102)$ & $63 \%(29)$ & $52.9 \%(9)$ & - \\
\hline Home PT & $13.9 \%(184)$ & $22.1 \%(40)$ & $13.5 \%(16)$ & $37 \%(17)$ & $41.2 \%(7)$ & - \\
\hline PAIR & $0.83 \%(11)$ & $0.5 \%(1)$ & 0 & 0 & $5.9 \%(1)$ & - \\
\hline Length of stay, ${ }^{\mathrm{b}} \mathrm{h}$ & 46.7 (SD, 45.0-8.3) & 46.7 (SE, 0.84) & 46.8 & 57.3 & 75.9 & $<0.001^{\circ}$ \\
\hline 30-day hospital revisit, d yes & $24.3 \%(321)$ & $27 \%(49)$ & $25.4 \%(30)$ & $21.7 \%(10)$ & $52.9 \%(9)$ & 0.037 \\
\hline
\end{tabular}

recommendation that time under observation status count toward the Medicare requirement ${ }^{11}$ has not been accepted, ${ }^{12}$ in large part because further expansion of PAIR services likely would be unaffordable to Medicare under its payment structure. ${ }^{13}$ Given our finding that the need for PAIR likely is much higher than previously anticipated, Medicare policy makers should consider broadening access to PAIR while efforts are made to rein in expenditures through payment reform.

One potential area of cost savings is more judicious use of PT evaluation for observation patients, particularly given our finding that the majority of PT consultations resulted in no further recommendations. Efforts to triage PT consultations for appropriateness have had some success, though the literature is scant. ${ }^{14}$ To improve value for Medicare, healthcare systems, and patients, researchers should rigorously evaluate approaches that maximize appropriate use of PT services.

\section{Hospital Length of Stay}

Our cohort's mean hospital stay was longer than averages reported elsewhere, ${ }^{9}$ likely reflecting our selection of Medi- care patients rather than a general medicine population. ${ }^{6}$ However, our cohort's adjusted mean hospital stay was significantly longer for patients recommended for PAIR than for patients without PT needs. That out-of-pocket costs for observation patients increase dramatically as LOS goes past 48 hours $^{6}$ could have significant financial implications for Medicare beneficiaries.

\section{Return Visits}

Almost 25\% of our observation patients returned to hospital within 30 days. There was a significant trend toward increased rehospitalization among patients recommended for PAIR than among patients with no PT needs.

Policies related to PAIR for observation patients are rooted in the concern that expanded access to services will contribute to overuse of services and higher healthcare costs. ${ }^{15}$ However, patients who could have benefited from PAIR but were not covered also were at risk for increased healthcare use and costs. A recent study found that more than one fourth of observation patients with repeat observation stays 
accrued excessive financial liability. ${ }^{16}$ Researchers should determine more precisely how the cost of coverage for PAIR placement on an index observation admission compares with the cost of subsequent healthcare use potentially related to insufficient supportive care at home.

\section{Study Limitations}

Our results must be interpreted within the context of study limitations. First is the small sample size, particularly the subset of patients selected for detailed manual chart review. We were limited in our ability to calculate sample size prospectively because we were unaware of prior work that described the association between PT recommendation and outcomes among observation patients. However, post hoc analysis estimated that a sample size of 181 patients would have been needed to determine a statistically significant difference in 30-day hospital revisit between patients recommended for PAIR and patients with no PT needs with $80 \%$ power, which we achieved. Although there are significant limitations to post hoc sample size estimation, we consider our work hypothesis-generating and hope it will lead to larger studies.

We could not account for the potential bias of the physical therapists, whose evaluations could have been influenced by knowledge of patients' observation status. Our findings could have underestimated the proportion of patients who otherwise would have been recommended for PAIR. Alternatively, therapists could have inaccurately assessed and overstated the need for PAIR. Although we could not account for the therapists' accuracy and biases, their assessments provided crucial information beyond what was previously obtained from administrative data alone. ${ }^{7,9}$

Hospital revisits were only accounted for within our hospital system - another potential source of underestimated findings. A significant proportion of patients recommended for home PT were discharged without services, which is counterintuitive, as Medicare covers home nursing services for observation patients. This finding most likely reflects administrative error but probably merits further evaluation.

Last, causality cannot be inferred from the results of a retrospective observational study.

\section{CONCLUSION}

As our study results suggest, there is an unmet need for PAIR services for Medicare observation patients, and LOS and subsequent use may be increased among patients recommended for PAIR. Our estimates are conservative and may underestimate the true need for services within this population. Our findings bolster MedPAC recommendations to amend the policies for Medicare coverage of PAIR services for observation patients.

\section{Acknowledgments}

The authors thank Paul Kolm, PhD, for statistical support.
Disclosures: Dr. Schwartz reports receiving personal fees from the Agency for Health Research and Quality, Bayer, the Blue Cross Blue Shield Association, Pfizer, and Takeda, all outside the submitted work. Dr. Hicks is supported by an Institutional Development Award from the National Institute of General Medical Sciences of the National Institutes of Health (grant U54-GM104941; principal investigator Stuart Binder-Macleod, PT, PhD, FAPTA). The other authors have nothing to report.

\section{References}

1. Ortman JM, Velkoff VA, Hogan H. An Aging Nation: The Older Population in the United States (Current Population Reports, P25-1140). Washington, DC: US Census Bureau; 2014. https://www.census.gov/prod/2014pubs/p25-1140.pdf. Published May 2014. Accessed January 1, 2016.

2. Carter C, Garrett B, Wissoker D. The Need to Reform Medicare's Payments to Skilled Nursing Facilities Is as Strong as Ever. Washington, DC: Medicare Payment Advisory Commission \& Urban Institute; 2015. http://www.urban.org/sites/ default/files/publication/39036/2000072-The-Need-to-Reform-Medicare-Payments-to-SNF.pdf. Published January 2015. Accessed January 1, 2016.

3. Cassidy A. The two-midnight rule (Health Policy Brief). HealthAffairs website. http://healthaffairs.org/healthpolicybriefs/brief_pdfs/healthpolicybrief_133.pdf. Published January 22, 2015. Accessed January 1, 2016.

4. Sheehy AM, Caponi B, Gangireddy S, et al. Observation and inpatient status: clinical impact of the 2-midnight rule. J Hosp Med. 2014;9(4):203-209.

5. Wright S. Memorandum report: hospitals' use of observation stays and short inpatient stays for Medicare beneficiaries (OEI-02-12-00040). Washington, DC: US Dept of Health and Human Services, Office of Inspector General; 2013. https:// oig.hhs.gov/oei/reports/oei-02-12-00040.pdf. Published July 29, 2013. Accessed January 1, 2016.

6. Hockenberry JM, Mutter R, Barrett M, Parlato J, Ross MA. Factors associated with prolonged observation services stays and the impact of long stays on patient cost. Health Serv Res. 2014;49(3):893-909.

7. Feng Z, Jung HY, Wright B, Mor V. The origin and disposition of Medicare observation stays. Med Care. 2014;52(9):796-800.

8. Ross MA, Hockenberry JM, Mutter R, Barrett M, Wheatley M, Pitts SR. Protocol-driven emergency department observation units offer savings, shorter stays, and reduced admissions. Health Aff. 2013;32(12):2149-2156.

9. Sheehy AM, Graf B, Gangireddy S, et al. Hospitalized but not admitted: characteristics of patients with "observation status" at an academic medical center. JAMA Intern Med. 2013;173(21):1991-1998.

10. Centers for Medicare \& Medicaid Services. Medicare \& Your Hospital Benefits. https://www.medicare.gov/Pubs/pdf/11408.pdf. CMS Product 11408. Published 2014. Revised March 2016. Accessed February 6, 2017.

11. Medicare Payment Advisory Commission. Hospital short-stay policy issues. In: Report to the Congress: Medicare and the Health Care Delivery System. Washington, DC: Medicare Payment Advisory Commission; 2015:173-204. http:// www.medpac.gov/docs/default-source/reports/chapter-7-hospital-short-stay-policy-issues-june-2015-report-.pdf. Published June 2015. Accessed January 1, 2016.

12. Centers for Medicare \& Medicaid Services (CMS), HHS. Medicare program: hospital outpatient prospective payment and ambulatory surgical center payment systems and quality reporting programs; short inpatient hospital stays; transition for certain Medicare-dependent, small rural hospitals under the hospital inpatient prospective payment system; provider administrative appeals and judicial review. Final rule with comment period; final rule. Fed Regist. 2015;80(219):70297-70607.

13. Medicare Payment Advisory Commission. Skilled nursing facility services. In: Report to the Congress: Medicare Payment Policy. Washington, DC: Medicare Payment Advisory Commission; 2015:181-209. http://www.medpac.gov/docs/ default-source/reports/chapter-8-skilled-nursing-facility-services-march-2015-report-.pdf. Published March 2015. Accessed January 1, 2016.

14. Hobbs JA, Boysen JF, McGarry KA, Thompson JM, Nordrum JT. Development of a unique triage system for acute care physical therapy and occupational therapy services: an administrative case report. Phys Ther. 2010;90(10):1519-1529.

15. Centers for Medicare \& Medicaid Services (CMS), HHS. Medicare program; hospital inpatient prospective payment systems for acute care hospitals and the long-term care hospital prospective payment system and fiscal year 2014 rates; quality reporting requirements for specific providers; hospital conditions of participation; payment policies related to patient status. Final rules. Fed Regist. 2013;78(160):50495-51040

16. Kangovi S, Cafardi SG, Smith RA, Kulkarni R, Grande D. Patient financial responsibility for observation care. J Hosp Med. 2015;10(11):718-723. 